\title{
Dos formas de escepticismo semántico
}

\author{
Plinio Junqueira Smith
}

\section{I}

\section{ntroducción}

En las últimas décadas, sobre todo después de la interpretación ofrecida por Kripke, ${ }^{1}$ surgió una nueva forma de escepticismo asociada con el nombre de Wittgenstein. Se suele llamar a esa nueva forma de escepticismo: "escepticismo semántico". ${ }^{2}$ Normalmente, se presenta la especificidad del escepticismo semántico de la siguiente manera. Los escépticos, antiguos o modernos, dudaron solamente de nuestras capacidades para discernir el valor de verdad de las oraciones (la suspensión de juicio consiste precisamente en no atribuir a ninguna oración un valor de verdad), pero el escéptico semántico pone en cuestión el contenido y la inteligibilidad de las propias oraciones. Naturalmente, se disputa si Wittgenstein es realmente un escéptico semántico, como algunos, siguiendo a Kripke, pretenden. Muchos, si no la mayoría, lo niegan. Pero el así llamado escepticismo semántico no está asociado solamente al nombre de Wittgenstein. Una vez que se empezó a utilizar ese término, muchos otros filósofos han sido tildados también como escépticos

${ }^{1}$ Véase Saul Kripke, Wittgenstein: on rules and private language. Oxford, Basil Blackwell, 1982.

${ }^{2}$ Véase Colin McGinn, Wittgenstein on meaning: an interpretation and evaluation. Oxford/Cambridge, Basil Blackwell, 1984. "Kripke claims (on Wittgenstein's behalf) that these three replies to the semantic sceptic exhaust the possibilities..." (ibid., p. 64) o "This question has two parts, corresponding to the constitutuive and epistemological versions of semantic scepticism" (ibid., p. 70). También se habla de un "escepticismo acerca del significado", como se ve en Boghossian: "it is hard to see how a convincing meaning scepticism could be confined purely to the linguistic domain..." (Paul Boghossian, "The Rule-Following Considerations", en Mind, vol. 98, núm. 392. Oxford, Universidad de Oxford, octubre, 1989, p. 509), o en Klaus Puhl, ed., Meaning Scepticism. Berlín/Nueva York, Walter de Gruyter, 1991. 
semánticos, entre ellos Quine y Davidson, ${ }^{3}$ y naturalmente se discute hasta qué punto la visión de estos autores sobre el lenguaje es semejante a la visión de Wittgenstein o a la visón de Wittgenstein presentada por Kripke.

El escepticismo semántico ha sido definido, no sin alguna ambigüedad, con la tesis de que no hay hechos objetivos que determinen el significado. A esa tesis filosófica se le llamó "tesis de la no factualidad". ${ }^{4}$ De acuerdo con esa tesis, un escéptico semántico sostiene que no hay algo objetivo ("a fact of the matter") o que no hay un hecho ("a fact") en el mundo que torne significativa una palabra o una oración. Por ejemplo, podemos atribuir dos significados distintos (conejo o condición de conejo) a una oración ("gavagai"), y los dos significados son perfectamente compatibles con todas las conductas y con todas las disposiciones a comportarse por parte de los hablantes competentes que usan esa oración. Dadas todas esas conductas y disposiciones a actuar, no podemos saber si la oración es aplicada a un animal o a un estadio de un animal. De esa manera, el significado de la oración no es determinado por nada objetivo en el mundo. Alternativamente, el escéptico semántico podría decir que el significado de una palabra está asociado a la regla que gobierna su uso y, una vez que no hay un hecho o hechos en el mundo que permitan identificar una regla específica, esa palabra, por no poseer una regla de uso, no tiene significado. De una o de otra manera, habrá una especie de subdeterminación del significado del lenguaje por los hechos objetivos del mundo. Así, el escéptico semántico es usualmente visto como el filósofo que cuestiona que existan hechos o alguna cosa objetiva en el mundo que determinen el significado de las palabras y oraciones. Sin embargo, me parece conveniente distinguir entre dos formas de escepticismo semántico, en tanto que su alcance, sus argumentos críticos y sus soluciones son muy diferentes. Esa distinción quedará más clara en los análisis más abajo, pero la indicación del origen de esa distinción debe ser inmediata.

En lo que sigue mi intención será menos la de saber hasta que punto Kripke tiene razón en interpretar a Wittgenstein como un escéptico semántico que la de distinguir dos formas, o variantes, de escepticismo semántico, una de las cuales es de inspiración wittgensteiniana y la otra de inspiración quineana. ${ }^{5}$

3 Véase K. Puhl, op. cit., y E. Orlando, "Una crítica del escepticismo semántico”, en Luiz Henrique Dutra y Plinio Junqueira Smith, orgs., Ceticismo: perspectivas históricas e filosóficas. Florianópolis, NEL/UFSC, 2000.

4 "Quine and Kripke's Wittgenstein both present 'skeptical' arguments for the conclusion that there are no facts about meaning". "This is what Kripke calls Wittgenstein's 'sceptical paradox' -the thesis that there is nothing, no fact, that could constitute meaning one thing rather than another" (C. McGinn, op. cit., p. 61).

${ }^{5}$ De hecho, es una cuestión actual saber si Quine y Wittgenstein sostuvieron visiones filosóficas similares sobre la naturaleza del lenguaje. Véase Robert Arrington y 
En el primer caso, hablaré de un escepticismo semántico dialéctico y, en el segundo, de escepticismo semántico científico, por razones que veremos en seguida. La perspectiva adoptada aquí será la perspectiva wittgensteiniana, tal y como la presenta Kripke. De hecho, inspirándose en Wittgenstein, Kripke hace una serie de breves, pero significativas observaciones sobre la filosofía del lenguaje de Quine. El presente texto es una especie de desarrollo de esas observaciones de Kripke sobre Quine en las cuales Kripke destaca algunas relaciones entre Quine y Wittgenstein. Algunos creen que esas observaciones apuntan hacia una aproximación de los dos filósofos. Me parece, sin embargo, que el tono general es crítico y que Kripke hace claras restricciones a las dudas planteadas por Quine sobre el significado.

En primer lugar, presentaré la forma quineana, o científica, de escepticismo semántico, mostrando algunas de sus ventajas. En seguida, expondré las críticas formuladas por el escéptico semántico wittgensteiniano, o dialéctico, indicando cuáles críticas no son muy convincentes y cuál es, a mi juicio, la crítica más promisora. Finalmente, trazaré, con mayor precisión la distinción entre las dos formas de escepticismo semántico. Antes de empezar la discusión, cabe indicar brevemente las dos formas de escepticismo semántico.

De un lado, tenemos lo que se puede llamar escepticismo semántico científico. Esa forma de escepticismo tiene su origen en una ciencia empírica del lenguaje y, a partir de esa perspectiva, cuestiona sobretodo la noción de significado. Las oraciones pueden ser verdaderas o falsas y los usos ordinarios que hacemos de ellas son significativos. Pero la teoría filosófica que entiende el lenguaje utilizando la noción, o el concepto, de significado es inaceptable. Lo que está en juego es la explicación que se debe dar de nuestras prácticas lingüísticas. Una concepción científica del lenguaje terminaría por mostrar que la teoría que recurre a la noción de significado es una mala teoría. La noción misma de significado debe ser abandonada por cualquier concepción del lenguaje que se pretenda empírica. Tal concepción científica del lenguaje, asociada a críticas a ciertas nociones claves, como la de referencia, está presente, por ejemplo, en las obras de Quine y Davidson.

De otro lado, tenemos lo que se puede llamar escepticismo semántico dialéctico. Esa forma de escepticismo semántico no presupone la adopción previa de una postura empirista en la filosofía. Su carácter es más bien dialéctico, esto es, la naturaleza de sus argumentos es la de presuponer solamente lo que los adversarios también presuponen y hacer que sus propias presuposiciones destruyan las tesis de los adversarios. Se trata, por lo tanto, de hacer 1996. 
volver contra los dogmáticos su propio juego dogmático. El argumento escéptico no requiere nada del escéptico, sino aquello que los dogmáticos aceptan por libre voluntad. Así, el escepticismo semántico dialéctico tiene no solamente una orientación distinta de la del científico, sino también un alcance distinto. Su blanco no es tanto la noción de significado, sino solamente una determinada y dogmática concepción del significado que debería ser sustituida por otra concepción mejor. Si la determinada y dogmática concepción del significado es aceptada, entonces se seguirían conclusiones absurdas, como la de que el propio lenguaje, en su uso común y cotidiano, no tiene significado. El aspecto negativo y destructivo del escepticismo semántico dialéctico ha sido llamado "la paradoja escéptica". Y la nueva concepción del significado, que evita esas consecuencias desastrosas, fue llamada "la solución escéptica”. Fue Kripke quien propuso esa forma de escepticismo, atribuyéndola a Wittgenstein. Como hay dudas sobre si Wittgenstein de hecho sostuvo un tal escepticismo y como Kripke no lo propuso en su propio nombre, se suele hablar de Kripkenstein como el defensor de esa forma de escepticismo semántico.

\section{El escepticismo semántico científico}

El escepticismo semántico científico parece, a primera vista, la línea más promisora de escepticismo, una vez que parece evitar las consecuencias absurdas de la parte negativa y destructiva de la forma dialéctica. Suponer que no hay lenguaje, o que el lenguaje no tiene lenguaje, es, de hecho, una visión demasiado escéptica, quizás nihilista. Queremos entender precisamente en qué consiste esa práctica humana que es el lenguaje y una teoría empírica parece el mejor camino para lograrlo.

No es parte de mi intención demostrar que el lenguaje no tiene ninguna significación. Yo no niego que las palabras y las oraciones de las cuales nos servimos tengan un sentido, en el sentido corriente de "tener un sentido". El lenguaje ordinario se aplica a situaciones, provoca respuestas y, en esa misma medida, presenta una significación. ${ }^{6}$

6 "Il n'entre pas dans mon intention de démontrer que le langage ne présente aucune signification. Que les mots et les phrases dont on se sert, au sans courant 'd'avoir un sens', aient un sens, je n'en disconviens pas. Le langage ordinaire s'applique à des situations, provoque des réponses, et dans cette mesure même, présente une signification" (Willard Quine, "Le mythe de la signification", en La Philosophie Analytique. París, Cahiers de Royaumont, ed. Minuit, 1962, p. 139). 
El escéptico semántico científico acepta tranquilamente que nuestras palabras y oraciones son significativas en su uso cotidiano y no lo pretende negar en ningún momento. Ése es, precisamente, el dato a ser explicado. Y una ciencia empírica es el mejor camino de explicarlo.

El escéptico semántico científico, como vimos, cuestiona solamente la noción de significado en tanto una noción teórica y explicativa del fenómeno de las conductas lingüísticas humanas. El escéptico semántico científico tiene un blanco muy preciso, esto es, quiere rechazar una determinada concepción filosófica del significado, la cual tiene por lo menos dos aspectos principales. En primer lugar, el significado sería una entidad, en particular una entidad mental, y las palabras serían etiquetas. El significado sería, pues, alguna cosa y alguna cosa distinta de las palabras y oraciones que emitimos en nuestras vidas cotidianas; será también neutral e independiente respecto de los diversos lenguajes. Cada lenguaje tendría significado en la medida en que sus palabras y oraciones estuvieran asociadas a esas entidades independientes. ${ }^{7}$ En segundo lugar, esa concepción supone que los hablantes tienen un significado determinado en la mente cuando hablan y que, por lo tanto, entender una palabra o una oración equivale a aprehender el significado determinado que está en la mente del otro. A esa concepción filosófica del significado, Quine la llamó "el mito del museo", 8 y constituye, en efecto, una teoría según la cual el lenguaje es una especie de copia de la realidad.

"El mito del museo", con las doctrinas de que el significado es una entidad mental y de que el significado está determinado en la mente del hablante, puede ser caracterizado también como una "semántica mentalista". Tal semántica mentalista no resiste a la crítica de una ciencia empírica del lenguaje. En primer lugar, podemos preguntarnos si entender una palabra, una oración o un lenguaje consiste en aprehender un significado determinado que está presente en la mente del hablante. Parece que no.

El escéptico semántico científico nos invita a pensar un caso extremo, el del lingüista de campo, que traduce un lenguaje totalmente desconocido para nuestro lenguaje (el inglés o el español). El "significado" es precisamente aquello que se preserva en una traducción y, según la semántica mentalista, traducimos un significado que está determinado en la mente del hablante nativo

7 "Ce contre quoi je m'insurge plus particulièrement, c'est l'idée d'une identité ou d'une communauté de sens sous le signe, ou d'une théorie de la signification qui en ferait une sorte d'abstraction supra linguistique, dont les formes du langage serais le pendant, ou l'expression. En somme, c'est à la signification en tant qu'idée que j'en ai" (W. Quine, "Le mythe de la signification", en op. cit., p. 139).

${ }^{8}$ W. Quine, Ontological Relativity and other essays. Nueva York, Universidad de Columbia, 1969 , pp. 27-29. 
cuando él dice una oración. Ahora bien, hay buenas razones para creer que hay distintas maneras por las cuales se pueden traducir todas las oraciones emitidas por el nativo, que son compatibles con todo lo que podemos observar y que, sin embargo, son incompatibles entre sí. Los datos empíricos disponibles para el lingüista de campo no le permiten escoger un determinado manual de traducción entre los varios manuales posibles. Ésa es la llamada "tesis de la indeterminación de la traducción". Si esta tesis es correcta, entonces no hay una única traducción que sea la traducción correcta, ya que todos los manuales de traducción son compatibles con los hechos observados, tanto como con las disposiciones a actuar del nativo. ${ }^{9}$ Se sigue, entonces, que el significado no está determinado en la mente del nativo.

El semántico mentalista supone también que el significado es una entidad (mental), para el cual la palabra es una etiqueta. Las palabras se refieren a ciertos objetos o a ciertas entidades mentales y esos objetos o esas entidades constituyen el significado de las palabras. La referencia, o extensión, constituiría el aspecto central del significado de las palabras. Pero, una vez más, una ciencia empírica del lenguaje nos muestra que no podemos identificar precisamente cual es la referencia de las palabras o de las oraciones. Por ejemplo, si el nativo dice "gavagai" o asiente a la pregunta ¿"gavagai"? cuando pasa un conejo delante de él (y él lo ve y nosotros lo observamos viendo el conejo), entonces no sabemos si "gavagai" se refiere a "conejo", "partes de conejo", "conejidad sucediendo", etcétera. Esa tesis fue llamada "tesis de la inescrutabilidad de la referencia". ${ }^{10}$ Por consiguiente, el significado no es una entidad, mucho menos una entidad mental.

Para el escéptico semántico científico, la semántica mentalista debe ser sustituida por una semántica conductista. Esta última es, de hecho, la mejor manera para comprender y explicar el comportamiento lingüístico humano. En este sentido, el lenguaje debe ser entendido como "un complejo de disposiciones presentes para la conducta verbal". ${ }^{11}$ Los hechos observables de que disponemos para aprehender el significado del lenguaje son hechos comportamentales, son las conductas de los hablantes. Desde niños, observamos las conductas de nuestros semejantes, hacemos conjeturas sobre sus conductas, en particular sobre sus conductas lingüísticas, y aprendemos con ellos a hablar. No hay otros hechos disponibles para nuestro aprendizaje del lengua-

9 "Manuals for translating one language into another can be set up in divergent ways, all compatible with the totality of speech dispositions, yet incompatible with one another" (W. Quine, Word and Object. Cambridge, MIT, 1960, p. 27).

10 "Reference itself proves behaviorally inscrutable", "extension itself goes inscrutable" (W. Quine, Ontological Relativity and other essays, p. 35).

${ }^{11}$ W. Quine, Word and Object, p. 27. 
je. Si uno examina de cerca los argumentos del escéptico semántico científico contra la semántica mentalista, uno percibe que sus argumentos ya presuponen una tal concepción conductista del lenguaje. Respecto de la tesis de la indeterminación de la traducción eso era muy claro. En uno de los pasaje más conocidos, Quine afirmaba que "manuales para traducción de un lenguaje para otro pueden ser elaborados de maneras divergentes, todos ellos compatibles con la totalidad de disposiciones verbales, sin embargo, incompatibles unos con los otros". ${ }^{12}$ No menos claro era el caso de la tesis de la inescrutabilidad de la referencia, pues, como decía Quine, "la propia referencia se muestra conductísticamente inescrutable". ${ }^{13} \mathrm{El}$ escéptico semántico científico tiene, por lo tanto, una concepción conductista del lenguaje. ${ }^{14}$

Otra de las razones para no aceptar la semántica mentalista es que, según el escéptico semántico científico, se puede hablar de nuestra conducta lingüística, así como explicar nuestra conducta lingüística, sin mencionar esas supuestas entidades, los significados. Cuando uno desarrolla una ciencia empírica para explicar el lenguaje humano, no hay ninguna necesidad de referirse a esas entidades dudosas. En otros términos, una ciencia empírica del lenguaje nos mostraría que los significados son postulaciones teóricas dispensables, o que los significados son conceptos teóricos sin contenido empírico claro.

De lo precedente, se percibe que el escepticismo semántico es un resultado de la práctica rigurosa de una ciencia empírica acerca del lenguaje. Las dudas respecto del significado se basan en una concepción conductista del lenguaje y la explicación positiva del lenguaje que el escéptico semántico nos ofrece no utiliza, en ningún momento, la noción de significado. Normalmente se supone que el escéptico es un enemigo de todo conocimiento, incluso del conocimiento científico. Pero vemos ahora que no siempre ese es el caso. ${ }^{15}$ La ciencia empírica nos lleva a sospechar de entidades metafísicas, a ver su aspecto dudoso y a percibir que hay un límite, aunque no siempre muy claro y bien definido, más allá del cual nuestras teorías pierden su contenido cognitivo y quizás su propia inteligibilidad. La perspectiva conductista del lenguaje nos permite, así, cuestionar una serie de conceptos filosóficos tradicionales, entre los cuales se destaca el de significado, como conceptos sumamente problemáticos. Para entender y explicar el lenguaje, basta hablar de

12 Véase nota 9.

13 Véase nota 10.

14 Para el caso de Davidson, véase E. Orlando, "Una crítica del escepticismo semántico", en op. cit.

15 Ese tampoco era el caso de Hume. Véase P. J. Smith, O ceticismo de Hume. São Paulo, Loyola, 1995, parte 2, cap. 3. 
disposiciones, asentimientos, reacciones en tales y tales circunstancias, etcétera. Esa explicación científica es precisamente aquella explicación que nos lleva a una forma de escepticismo semántico.

\section{Críticas del escéptico semántico dialéctico al escepticismo semántico científico}

La forma científica del escepticismo semántico, sin embargo, no estuvo exenta de críticas provenientes de todas las partes, incluso de la forma dialéctica del escepticismo semántico. Son las objeciones del escéptico semántico de inspiración wittgensteiniana las que nos interesan ahora. Mi intención es no solamente exponerlas, para marcar las distancias entre las dos formas de escepticismo semántico, sino de discutirlas también, aunque no con muchos detalles, para evaluarlas mínimamente.

En primer lugar, como quedó obvio en el item anterior, la forma científica del escepticismo semántico presupone una perspectiva conductista. Eso, en sí mismo, no es un problema, pues uno puede aceptar el conductismo si le parece la postura correcta para entender el ser humano en sus más variadas actividades y prácticas. Pero la dependencia del escepticismo semántico de una determinada teoría psicológica seguramente lo debilita, ya que ahora toda la cuestión depende, no tanto de consideraciones lógico-semánticas respecto de la noción de significado, sino más bien en consideraciones de otro orden. Apoyarse en el conductismo trae, a los ojos de mucha gente, problemas insolubles y dificultades insuperables, además de una demasiado vieja doctrina psicológica. De hecho, se dijo que la metodología conductista, aun cuando Quine la defendió hace muchas décadas, ya estaba superada y era, probablemente, muy limitada en alcance. ${ }^{16}$ Hay problemas no solamente con su metodología, sino también con la base empírica del conductismo. Wittgenstein, si Hacker tiene razón, ${ }^{17}$ no aceptaría jamás la metodología conductista para comprender el fenómeno del lenguaje humano. De hecho, el propio Wittgenstein

16 Después de afirmar que el lingüista de campo de Quine debe "adopter une méthode que je croyais pour ma part depuis longtemps passé de mode en psychologie, et qui est d'étudier les réactions à une excitation", concludes, in a not very elegant way, to characterize him (the field linguist, of course) as "un des plus fameux imbéciles que j'aie jamais rencontré" (G. Ryle, "Le mythe de la signification", en La Philosophie Analytique. París, Cahiers de Royaumont, ed. Minuit, 1962, p. 181).

${ }^{17}$ Hacker, por ejemplo, afirma que Wittgenstein "would reject Quine's behaviorist methodology" (P. M. S. Hacker, Wittgenstein's Place in the Twentieth-Century Analytic Philosophy. Oxford, Blackwell, 1996, p. 220; también pp. 218-223). 
anticipó que su pensamiento pudiera ser confundido con el conductismo y se alejó explícitamente de tal sugerencia, de tal modo que su propio testimonio ya indica que una lectura conductista de las Investigaciones filosóficas deja escapar el pensamiento wittgensteiniano. ${ }^{18}$ ¿Por qué uno debería aceptar que una ciencia empírica del lenguaje humano debería tener como su base empírica una conducta descrita como alguna cosa puramente física, como un estímulo nervioso, o como un movimiento físico (no como una acción)? Esa parece ser una elección arbitraria de una base empírica para una ciencia. ${ }^{19} \mathrm{Si}$ tenemos que aceptar el conductismo para podernos plantear nuestras dudas escépticas respecto de la noción de significado, entonces no hay tanto peligro en que nos tornáramos escépticos, pues el conductismo, aunque tenga todavía muchos adeptos, seguramente no tiene el mismo prestigio de otras épocas, ni está exento de innumerables críticas.

Naturalmente, el conductismo no es una elección arbitraria de Quine y, en el caso del lenguaje, sobre todo en el caso del aprendizaje del lenguaje, el conductismo parece estar en una posición particularmente cómoda, pues ¿qué más hay para aprender el lenguaje sino la observación de la conducta ajena? Si un niño no sabe todavía hablar el español, él lo aprenderá escuchando lo que le dicen, mirando lo que le hacen mirar, percibiendo lo que hacen los otros, etcétera. Esto es todo lo que el niño dispone para, poco a poco, aprender el lenguaje. Por cierto, tiene el niño ciertas capacidades naturales como la de escuchar, la de reproducir sonidos, la de distinguir colores, etcétera, que le permiten aprender por observación. Respecto de su relación con sus padres, hermanos, maestros y demás seres humanos, todo a lo que él tiene acceso son solamente conductas, como los demás pueden meramente observar la conducta del niño aprobándola o censurándola para aprender correctamente el español. Muchos filósofos y lingüistas, en particular muchos wittgensteinianos, no aceptan la idea de que el conductismo es la única manera de entender y describir el aprendizaje de una lengua por parte del niño. Glock, por ejemplo, reconoce que la fuerza de la posición de Quine se basa en la idea de que la perspectiva conductista parece obligatoria, porque para aprender un lenguaje dependemos estrictamente de la conducta en situaciones observables. Pero, añade él, "a pesar de no existir alternativa para aprender el lenguaje del nati-

18 Otra interpretación para la cual Wittgenstein se aleja del conductismo se encuentra en E. Tugendhat, Selbstbewusstsein und Selbstbestimmung. Fráncfort del Meno, Suhrkamp, 1979, cap. 5.

19 Véase, por ejemplo, la crítica de Orlando a la doctrina de la interpretación radical de Davidson, o las objeciones de Stroud a las perspectivas de Quine sobre el significado. (E. Orlando, "Una crítica del escepticismo semántico", en op. cit., pp. 24-32 y Barry Stroud, Meaning, Understanding and Practice: Philosophical Essays. Oxford, Universidad de Oxford, 2000). 
vo, sino con base en lo que ellos dicen y hacen, hay una alternativa para describir lo que ellos dicen y hacen en el idioma conductista de estímulo y respuesta, una alternativa a ser encontrada en Wittgenstein". No es posible, ni es el caso, de discutir el conductismo aquí, sino solamente de apuntar el hecho de que hacer el escepticismo semántico depender del conductismo torna esa forma de escepticismo semántico menos fuerte y, por lo tanto, menos atractivo. Más adelante, me referiré a la idea general a ser encontrada en Wittgenstein. ${ }^{20}$ De cualquier manera, tal consideración no resulta, en sí misma, un rechazo, ni del conductismo ni del escepticismo semántico.

Otro argumento utilizado contra el escéptico semántico científico es una reductio ad absurdum. Si los argumentos contra las nociones de referencia y de significado son correctos, esto es, si las tesis de la inescrutabilidad de la referencia y de la indeterminación de la traducción son correctas, entonces nosotros no somos capaces de distinguir entre dos referentes distintos, como conejo, parte de conejo, o condición de conejo, etcétera. Si aceptamos la explicación conductista del lenguaje, entonces deberíamos aceptar esa conclusión absurda, según la cual somos incapaces de discriminar lo que de hecho discriminamos. Cualquier teoría del significado debería ser capaz de distinguir entre una palabra (u oración) que significa, o se refiere, a un animal y una palabra (u oración) que se refiere a una parte o una condición de ese animal. Una vez que la teoría conductista no puede hacer eso (por su propia confesión), se sigue que es absurda e inaceptable.

Esa objeción, sin embargo, es mala, porque la doctrina del escéptico semántico científico no es la de que no podemos distinguir entre distintos referentes, sino la de que no hay una cuestión "absoluta" sobre el referente, independiente de un lenguaje particular. Si aceptamos hablar siempre teniendo en cuenta un lenguaje particular, entonces podemos seguramente distinguir

20 "His [Quine's] rationale is that 'the behaviorist approach is mandatory', because in learning a language 'we depend strictly on overt behaviour in observable situations'. But although there is no alternative to learning the native's language on the basis of what they say and do, there is an alternative to describing what they say and do in the behaviourist idiom of stimulus and response, an alternative to be found in Wittgenstein" (H.-J. Glock, "On safari with Wittgenstein, Quine and Davidson", en R. Arrington y H.J. Glock, eds., Wittgenstein and Quine, p. 149). Ideas similares son desarrolladas por Shanker (S. Shanker, "The conflicts between Wittgenstein and Quine on the nature of language and cognition and its implications for constraint theory", en R. Arrington y H.-J. Glock, eds., Wittgenstein and Quine). Por otro lado, quizás se pueda decir que Davidson no es un conductista. Como es bien conocido, su base empírica es diferente de la de Quine. Pero también sobre esto se disputa. Véase E. Orlando, "Una crítica del escepticismo semántico", en op. cit., y H.-J. Glock, "On safari with Wittgenstein, Quine and Davidson", en op. cit., pp. 156-163). 
entre los referentes para ese lenguaje. Si nuestro lenguaje es el español (o el inglés), tenemos todo un "aparato de individuación", constituido por "pronombres, plurales, identidades, numerales, etcétera", por medio del cual distinguimos entre un conejo y sus partes o sus estados. "Dado ese aparato, no hay misterio sobre la extensión; los términos tienen la misma extensión cuando son verdaderos de las mismas cosas". ${ }^{21}$ Obviamente, "conejo", "parte de conejo" y "condición de conejo" no son verdaderos de las mismas cosas. Las consecuencias absurdas de las cuales habla la objeción en el párrafo precedente solamente se siguen si uno acepta la noción de que el significado es independiente de cualquier lenguaje, o si se supone que la cuestión del significado y del referente se ponen sin hablar de un lenguaje particular. Por eso mismo, sostiene el escéptico semántico, tenemos que parar de hablar como si una cuestión, planteada de manera absoluta, tuviera sentido.

Hay, de acuerdo con otra queja del escéptico semántico de inspiración wittgensteiniana, una limitación bastante grande en la perspectiva conductista del escéptico semántico cientificista, a saber, la de que el lenguaje debe ser entendido desde un punto de vista externo, esto es, para entender el significado nosotros debemos observar la conducta de otra persona. El significado no sería alguna cosa determinada que está en la cabeza o en la mente de alguien y que sería aprehendido por el que escucha, como supone el semántico mentalista, sino que sería una especie de traducción o de interpretación del que escucha. Observando la conducta de otra persona, el lingüista de campo, o el niño, o nosotros mismos, hacemos una hipótesis sobre su conducta lingüistica y postulamos un significado para la cadena sonora que escuchamos. Toda la cuestión es la de hacer sentido de las conductas ajenas, todo el problema del significado se pone como el problema de traducir o interpretar una cadena sonora emitida por otra persona. Atribuimos significado a la cadena sonora, emitida por otra persona, basados en la observación de su conducta, de su entorno y de su observación de su entorno. El significado no es alguna cosa dada en la cadena sonora, sino más bien lo que nuestra traducción o interpretación le atribuye como una hipótesis empírica plausible. Quizás, si tuviéramos otro punto de vista sobre el lenguaje, el escepticismo semántico,

${ }^{21}$ W. Quine, Ontological Relativity and other essays, p. 35. "Fair enough; reference is nonsense except relative to a coordinate system. In this principle of relativity lies the resolution of our quandary... It is meaningless to ask this absolutely; we can meaningfully ask it only relative to some background language" (ibid., p. 48). "What makes ontological questions meaningless when taken absolutely is not universality but circularity. A question of the form 'What is an F?' can be answered only by recourso to a further term: 'An F is a G'. The answer makes only relative sense: sense relative to the uncritical acceptance of 'G'” (ibid., p. 53). 
por lo menos en esa forma, seria evitable. Si, por ejemplo, pensáramos en el lenguaje a partir de mi propio caso, a partir de lo que yo quiero decir ahora, o quise decir en el pasado, con tal y tal expresión, entonces es posible que la noción de significado no sea tan problemática cuanto se quiere hacerla. Una perspectiva de primera persona, y no de tercera persona, podría, quizás, resistir a la crítica del escéptico semántico cientificista. El significado no sería lo que atribuimos a los otros, como una especie de traducción o interpretación, pero alguna cosa experimentada por nosotros mismos. ${ }^{22}$

¿Qué pensar de esa queja? Que es, una vez más, injusta. Me parece falso decir que el escéptico semántico científico excluye el "yo" de sus consideraciones, como si estuviera restringido a consideraciones de tercera persona. El "yo" seguramente hace parte de las consideraciones del lenguaje, pero no juega ningún papel privilegiado, como el propio wittgensteiniano lo admitirá. El recurso al lingüista de campo que hace la traducción de una lengua totalmente desconocida para el español (o inglés) era un mero recurso: "la traducción radical empieza en casa". ${ }^{23}$ "En casa" quiere decir no sólo con mis vecinos que hablan la misma lengua que yo, en cuyo caso yo tengo que usar una regla de traducción homofónica para entenderlos, pero yo también, en mi propio caso, tuve que aprender a hablar el español y mis padres y profesores me lo enseñaron observándome y corrigiéndome. El "yo" es uno entre otros y no tiene un lugar privilegiado, pues, "como enfatizó Dewey, no hay un lenguaje privado". ${ }^{24}$ Sería un error suponer que el "yo" es una especie de punto focal a partir del cual se pueden reconstruir todas las conductas lingüísticas de los demás seres humanos. El "background language" a partir del cual se pueden plantear los problemas semánticos no es un "lenguaje privado", es un lenguaje como el español o el inglés. En segundo lugar, no parece muy claro qué sería esa "experiencia subjetiva" que uno tendría del significado, que sería aprehendida solamente desde la perspectiva de la primera persona, pero no desde la perspectiva de la tercera persona. Resulta muy raro un filósofo de inspiración wittgensteiniana apelar a una tan dudosa (y mentalista) noción.

Finalmente, llegamos a aquel argumento que parece ser el más fuerte que se dirige contra el escéptico semántico científico. Una ciencia empírica acerca del lenguaje, éste es el nudo de la cuestión según el escéptico semántico de inspiración wittgensteiniana, solamente describe nuestras disposiciones para actuar y reaccionar de ciertas maneras, sin ser capaz de explicar el elemento

${ }^{22}$ Véase S. Kripke, op. cit., pp. 14-15 y C. Wright, "Kripke's account of the argument against private language", en The Journal of Philosophy, vol. LXXXI, 1984, pp. 289-305.

${ }^{23}$ W. Quine, Ontological Relativity and other essays, p. 46.

${ }^{24}$ Ibid., p. 47. 
esencial del lenguaje, a saber, su normatividad. ${ }^{25}$ Cualquier teoría del comportamiento humano lingüístico que no es capaz de explicar sus normas, o las reglas por las cuales es gobernado, no puede ser una teoría satisfactoria. Si nuestra teoría no lleva en consideración el aspecto normativo del lenguaje, entonces lo que está siendo explicado no es el lenguaje, pues el lenguaje (y el significado) es esencialmente normativo. El objeto en consideración es simplemente ignorado o abandonado. Hoy en día parece un punto universalmente aceptado que la explicación de la normatividad es un requisito básico de cualquier teoría sobre el lenguaje. Es una observación trivial la de que si yo sé el significado de una palabra, entonces yo sé en cuales casos es correcto usarla y en cuales casos es incorrecto usarla. ${ }^{26}$ Ya no es tan trivial sostener que podemos distinguir los casos correctos de los incorrectos por medio de una regla que gobierne el uso de las palabras. La regla para una palabra determina lo que es correcto decir en una cierta circunstancia, esto es, determina lo que yo debo decir si yo uso la palabra correctamente, esto es, de acuerdo con la regla. Sin embargo, una explicación conductista del significado explica solamente por qué yo estoy dispuesto a decir tal y tal cosa en tal y tal circunstancia, o a predecir (con buen grado de certeza) que yo diré tal y tal cosa en tal y tal circunstancia, pero no lo que yo debo decir. El conductista meramente describe nuestras reacciones lingüísticas, pero no intenta entender por qué algunas de ellas son correctas o justificadas, mientras que otras no lo son.

Otra formulación de ese problema es la siguiente. Para el escéptico semántico científico, la base empírica de su teoría es el asentimiento (o disentimiento) de una aserción frente a ciertas circunstancias del mundo observable. Si la oración es (o le parece) verdadera, el nativo da su asentimiento; si es (o le parece) falsa, no le da su asentimiento. Pero la idea de que el lenguaje es una práctica o una actividad normativa presupone, o implica, más que eso. Para describir esa actividad, necesitamos hacer referencias a ciertas prácticas

25 "The dispositionalist gives a descriptive account of this relation: if '+' meant addition, then I will answer ' 125 '. But this is not the proper account of the relation, which is normative, not descriptive... The relation of meaning and intention to future action is normative, not descriptive" (S. Kripke, op. cit., p. 37). Véase Hacker: "From the point of view of a normative (rule-governed) conception of meaning and language such as Wittgenstein defends, a behaviourist conception of Quine's is simply no conception of meaning at all, not even as ersatz one. Indeed, it is no conception of language, for a language stripped of normativity is no more language than chesse stripped of its rules is a game" (P. M. S. Hacker, Wittgenstein's Place in the TwentiethCentury Analytic Philosophy, p. 211).

26 "The normativity of meaning turns out to be, in other words, simply a new name for the familiar fact that, regardless of whether one thinks of meaning in truth-theoretic or assertion-theoretic terms, meaningful expressions possess conditions of correct use" (P. Boghossian, "The Rule-Following Considerations", en op. cit., p. 513). 
normativas, tales como la introducción, enseñanza y explicación de padrones de corrección, bien como críticas y aprobación de actuaciones con referencia a esos padrones de corrección. Desde la perspectiva wittgensteiniana, tal como la defendida por Glock, es necesario distinguir dos tipos de asentimiento o disentimiento. Hay no solamente el asentimiento o disentimiento apuntado por el escéptico semántico científico, sino también el asentimiento o disentimiento con referencia a los patrones de corrección. Una oración puede ser rechazada como incorrecta, sin sentido o absurda respecto de nuestros patrones de corrección. "Una práctica", añade Glock, "sin esa distinción entre el falso y el sin sentido sería, en el mejor de los casos, un bla-bla-bla fonético comunitario (a communal phonetic babbling)". ${ }^{27}$ De esta manera, el escéptico semántico científico, limitado por su conductismo, está resignado a aceptar que los términos no tienen significado, negando el fenómeno mismo que quería explicar.

\section{La perspectiva del escéptico semántico dialéctico}

El escepticismo semántico dialéctico presenta una concepción del lenguaje muy distinta de la concepción conductista del escéptico semántico científico. El lenguaje, para el primero, es una actividad normativa, y no, como para el último, un conjunto de disposiciones para actuar verbalmente. Sus dudas escépticas respecto del lenguaje y de la noción filosófica del significado no se basan, o no presuponen, una previa aceptación del conductismo, ni de ninguna ciencia empírica en particular. La base empírica no está restringida, de ninguna manera, solamente a las conductas observables, sino que también se pueden invocar cualesquiera tipos de hechos, como los hechos mentales. La metodología también no se restringe a la mera observación de conductas o a preguntas al hablante, tomando en cuenta solamente si él asiente o disiente de la oración propuesta, y el escéptico semántico dialéctico puede aceptar incluso la controvertida noción de introspección, como la manera de acceder a nuestros estados mentales internos. No hay, a priori, o de manera dogmática, una exclusión de la observación interna; tal rechazo sólo se puede dar al final de una investigación. Además, el escéptico semántico dialéctico concede una memoria perfecta y una capacidad para conocer todos los hechos, como si fuéramos dioses, con el intento de mostrar que el problema es estrictamente lógico-semántico, y no epistemológico. Desde el punto de vista epistemológico,

${ }^{27}$ H.-J. Glock, "On safari with Wittgenstein, Quine and Davidson", en op. cit., p. 163. Glock cree que esas críticas valen tanto para Quine como para Davidson. 
se admite acceso a todos los tipos de hechos y un conocimiento perfecto de esos hechos. Así, se puede combinar tanto una perspectiva externalista, cuanto una internalista, pues el problema escéptico se formula de manera neutral respecto de perspectivas. La paradoja escéptica no depende de ninguna perspectiva epistemológica o científica acerca del significado.

Si, para el escéptico semántico científico, la filosofía era solo una ciencia empírica de carácter más general, para el escéptico semántico dialéctico, la filosofía no es, de ninguna manera, una ciencia empírica, pero, al revés, consiste en un análisis conceptual a priori, en el sentido de que sus consideraciones y argumentos no dependen de ningún descubrimiento nuevo resultante de la investigación empírica. El escepticismo semántico, desde esta perspectiva, no es resultado de una ciencia empírica, pero de argumentos de un tipo muy diferente. El argumento escéptico de esta segunda forma es, para decirlo brevemente, una forma de reductio ad absurdum. Si concebimos el lenguaje de una cierta manera, entonces la conclusión será la de que no hay tal cosa como un lenguaje. ${ }^{28} \mathrm{Si}$ aceptamos que el lenguaje tiene significado, según una cierta concepción de significado, entonces debemos aceptar que el lenguaje no tiene significado. Es una reducción al absurdo porque una determinada teoría semántica lleva a una cierta conclusión absurda o, en términos más estrictos, porque la conclusión niega lo que aceptamos en la premisa. Es un argumento dialéctico porque el escéptico semántico no pide ninguna premisa que ya no sea aceptada y endosada por el filósofo dogmático. Se trata de extraer, de las propias doctrinas defendidas por los filósofos dogmáticos, consecuencias inaceptables que los obligan a abandonar lo que inicialmente aceptaban. Esa estrategia parece ser la más fuerte, ya que ella no acepta arbitrariamente lo que mucha gente no acepta, como el conductismo, y, haciendo exactamente lo opuesto, acepta solamente lo que su oponente acepta, argumentando a partir de las presuposiciones de este último.

El escéptico semántico dialéctico, por otro lado, no solamente reconoce, pero llama la atención para el aspecto normativo del lenguaje. Su paradoja es construida precisamente sobre ese aspecto. Él no plantea un problema específicamente sobre la referencia, o sobre la intención del hablante, sino sobre las reglas que gobiernan el uso correcto del lenguaje. Justamente lo que el escéptico semántico científico no explicaba satisfactoriamente, o quizás ni siquiera intentaba explicar, es, para el escéptico semántico dialéctico, el nudo de toda la cuestión. Como vimos, el entendimiento del significado de una palabra u oración involucra la capacidad de distinguir entre el uso correcto y

28 "But before we pull the rug out from under our own feet..." (S. Kripke, op. cit., p. 13) y "The ladder must finally be kicked away" (ibid., p. 21). 
el uso incorrecto. Sin esa distinción, el lenguaje es sin sentido. Las reglas semánticas son lo que nos permite trazar esa distinción, de modo que el escéptico semántico dialéctico propondrá su reto con respecto a ellas: ¿hay tales reglas?, ¿están las reglas determinadas por algún hecho en el mundo? Si no hay tales reglas semánticas, el lenguaje está desprovisto de significado. ${ }^{29}$

Quizás sea importante insistir en el último punto, el más importante, en tanto concierne al problema planteado por el escéptico semántico dialéctico, que es diferente del problema planteado por el escéptico semántico científico. El problema planteado por el escéptico semántico científico es un problema sobre la noción de significado como una entidad más allá de los lenguajes, una entidad externa a los lenguajes que darían a estos su significado. Una vez que no podemos fijar sus referentes, esta noción de significado seria inútil y lo mejor seria pura y simplemente no hablar más de significado. Pero el problema planteado por el escéptico semántico dialéctico es enteramente diferente. No es un problema sobre el significado entendido como una entidad externa al lenguaje, pero es un problema sobre las reglas que gobiernan los usos del lenguaje. Cuando usamos una palabra con significado, la usamos de manera correcta en determinada circunstancia. Usar la palabra es un hecho que ocurre en el mundo, en un determinado lugar y en un determinado tiempo. Si usamos la palabra correctamente, es alguna cosa que depende de la regla de uso de esa palabra: si la usamos de acuerdo con la regla, entonces la usamos correctamente; y si no la usamos de acuerdo con la regla, entonces la usamos incorrectamente. Toda la paradoja del escéptico semántico dialéctico es la de saber si los hechos en el mundo, esto es, lo que yo hice, lo que yo dije, lo que yo pensé, etcétera, permiten identificar una única regla que me lleve a distinguir entre los usos correctos y los usos incorrectos de la palabra. Si hay una regla que gobierna el uso de la palabra, entonces hay usos correctos e incorrectos; pero, si no hay tal regla, entonces la palabra carece de significado y no pasa de un mero ruido. Por ejemplo, si hay una regla para el uso de "mesa" o de "verde" y si yo conozco esa regla, entonces hay usos correctos e incorrectos y yo sé cuales son esos usos. No hay, para el escéptico semántico dialéctico, ningún problema en la aplicación de la regla. ${ }^{30}$ El problema está

29 "Having a meaning is essentially a matter of possessing a correctness condition. And the sceptical challenge is to explain how anything could possess that" ( $P$. Boghossian, "The Rule-Following Considerations", en op. cit., p. 515).

${ }^{30}$ El problema de la paradoja rechazada por Wittgenstein parece ser el de la aplicación de la regla: si, para saber cual es el uso correcto, yo necesito de una interpretación de la regla, entonces no puedo jamás saber eso. El problema de la paradoja de Wittgenstein, pues, no es el mismo problema que el de la paradoja escéptica presentada por Kripke. 
situado entre los hechos (pasados y presentes, físicos y mentales, individuales y sociales, etcétera) y la regla: ¿̇ónde está el vínculo que permite a los hechos de cualquier tipo identificar una única regla que gobierne el uso de las palabras o del lenguaje? La paradoja escéptica muestra, o pretende mostrar, que, si tenemos una concepción realista clásica del significado, esto es, si concebimos el significado a partir de una semántica de condiciones de verdad, entonces no hay cómo entender o explicar la normatividad del lenguaje.

Toda la cuestión sería, ahora, examinar la concepción normativa del lenguaje en sus diversas variantes, tanto en su aspecto negativo como en el positivo. Por ejemplo, ver cómo Wittgenstein rechaza ciertas concepciones semánticas mentalistas para explicar las reglas que rigen el lenguaje como un mecanismo mental, que seria inaceptable; o cómo Wittgenstein aclara el papel de las normas en el funcionamiento del lenguaje, si por una supuesta "relación interna" ${ }^{31}$ o no. También se debería investigar cuidadosamente la "paradoja escéptica" propuesta por Kripkenstein, así como la "solución escéptica" y la "visión de la comunidad". Sin embargo, esta tarea ya nos llevaría demasiado lejos. Mi intención era solamente hacer una breve introducción al así llamado escepticismo semántico desde una perspectiva particular, la del escéptico semántico dialéctico.

31 Ésta es la interpretación de Baker y Hacker (G. Baker y P. M. S. Hacker, Scepticism, Rules and Language. Oxford, Basil Blackwell, 1984). 